\title{
Continuous Venovenous Hemodiafiltration Versus Standard Medical Therapy for the Prevention of Rhabdomyolysis-Induced Acute Kidney Injury: A Retrospective Cohort Study
}

\section{Yan Meng ( $\sim$ mbao124@qq.com )}

The Affiliated Hospital of Inner Mongolia Medical University https://orcid.org/0000-0002-9236-5546

\section{Ming-xue Zhou}

The Affiliated Hospital of Inner Mongolia Medical University

Chun-bo Wu

The Affiliated Hospital of Inner Mongolia Medical University

\section{Dehua Wang}

The Fifth Hospital of Shijiazhaung

\section{Dong-yin Shi}

The Affiliated Hospital of Inner Mongolia Medical University

Jian-rong Zhao

The Affiliated Hospital of Inner Mongolia Medical University

\section{Research}

Keywords: Continuous renal replacement therapy (CRRT), Continuous venovenous hemodiafiltration (CVVHDF), Rhabdomyolysis, Acute kidney injury, Health economics

Posted Date: November 18th, 2021

DOI: https://doi.org/10.21203/rs.3.rs-1055633/v1

License: (c) (i) This work is licensed under a Creative Commons Attribution 4.0 International License. Read Full License 


\section{Abstract}

Aim: To determine whether continuous venovenous hemodiafiltration (CVVHDF) plus standard medical therapy (SMT) vs. SMT alone prevents rhabdomyolysis (RM)-induced acute kidney injury (AKI) and to analyze the related health economics.

Methods: This retrospective cohort study involved patients with RM without AKI, coronary heart disease, or chronic kidney disease treated with CVVHDF plus SMT (treatment group, $n=9$ ). Matched patients with RM without AKI treated with SMT only served as controls (1:1 ratio). Baseline characteristics, biochemical indexes, renal survival data, and health economic data were compared between groups. In the treatment group, some biochemical data were compared at different time points.

Results: At 2 and 7 days after admission, serum biochemical indices (e.g., myoglobin, creatine kinase, creatinine, and blood urea nitrogen) did not differ between the two groups. Total $(P=0.011)$ and daily hospitalization costs $(P=0.002)$ were higher in the treatment group than in the control group. After 53 months of follow-up, no patient developed increased serum creatinine, except for 1 treatment-group patient who died of acute myocardial infarction. In the treatment group, myoglobin levels significantly differed before and after the first CVVHDF treatment $(P=0.008)$, and serum myoglobin, serum creatinine, and blood urea nitrogen decreased significantly at different time points after CVVHDF.

Conclusions: Although CVVHDF facilitated myoglobin elimination, its addition to SMT did not improve serum myoglobin or other biochemical indices or the long-term renal prognosis. Despite similar hospitalization durations, both total and daily hospitalization costs were higher in the treatment group.

\section{Introduction}

Rhabdomyolysis (RM) is a clinical syndrome characterized by the injury and disintegration of skeletal muscle fibers followed by the release of myoglobin, electrolytes, enzymes, and other components of the injured skeletal muscle cells into the blood circulation[1, 2]. RM can be caused by a variety of traumatic and non-traumatic factors, such as excessive exercise, crush injuries, statins, poisoning, infections, and autoimmune diseases. The main clinical manifestations of RM are muscle pain, weakness, and dark teacolored urine. Mild RM may be asymptomatic, but as the creatine kinase level rises beyond a certain threshold, severe complications such as electrolyte imbalance/disturbances, acute kidney injury (AKI), and disseminated intravascular coagulation may occur[3]. AKI is the most common and most severe complication of RM, and is reported to occur in $10-60 \%$ of patients with RM[4]. The occurrence of AKI can significantly worsen the prognosis of patients with RM, and the reported mortality rates of RM patients who develop AKI range from 7-80\%[5]. Among RM patients in the intensive care unit, the mortality rate has been reported to be $59 \%$ when AKI was present and $22 \%$ when it was not[6].

In addition to conventional drug therapy, renal replacement therapy (RRT), including continuous or intermittent hemodialysis, hemofiltration, and hemodiafiltration, plays an important role in the treatment of RM-induced AKI. For patients with AKI, especially when associated with life-threatening complications 
such as hyperkalemia, hypercalcemia, hyperazotemia, anuria, or hyperhydration, RRT is indispensable[7]. RRT can remove uremic toxins and excess water, regulate the stability of the internal environment, and create a good environment for the recovery of renal and other organ functions. However, it is unclear whether RRT can prevent the occurrence of AKI in patients with RM. Although it has been reported that hemofiltration can effectively remove a certain amount of myoglobin (molecular weight, $17 \mathrm{kDa}$ ) from the blood of patients with RM[8,9], most of these studies were case reports without control groups, and did not use the recovery of renal function and long-term prognosis as the study end-points. Upon searching the literature, we found no well-designed cohort study or randomized controlled trial probing the preventive effects of hemofiltration or hemodiafiltration on RM-induced AKI. Therefore, in this retrospective cohort study, we compared the effectiveness of continuous venovenous hemodiafiltration (CVVHDF) combined with standard medical therapy (SMT) versus SMT alone in preventing AKI in patients with RM. In addition, we conducted a health economic analysis of the two treatment arms.

\section{Materials And Methods}

\subsection{Study design and patient selection}

This retrospective single-center study involved patients who were diagnosed with RM and treated with CVVHDF in the Affiliated Hospital of Inner Mongolia Medical University, between April 2017 and December 2020. Patients with newly diagnosed coronary heart disease, AKI, or chronic kidney disease (CKD) were excluded. AKI was diagnosed using the 2012 Kidney Disease: Improving Global Outcomes diagnostic criteria[10]. The remaining patients who had RM but not AKI and were treated with CVVHDF combined with SMT formed the treatment group.

\subsection{CVVHDF and SMT}

Details of the CVVHDF procedure are presented in Table 1. Before the initiation of CVVHDF, vascular access was secured by the venous insertion of a 12-French two-lumen hemodialysis catheter. The number of CVVHDF sessions was determined by the physician-in-charge based on the assessment of the patient's condition. Each patient was hospitalized for approximately 1 week, and during this time, 1, 2, or 3 CVVHDF sessions were conducted. The treatment was administered by well-trained nurses using a Baxter Prismaflex system and an M150 set, with an effective surface area of $1.5 \mathrm{~m}^{2}$ and a myoglobinsieving coefficient of 0.70 . Low-molecular-weight heparin was used for anticoagulation. A single treatment session lasted for $10-22 \mathrm{~h}$. The dialysate flow rate was set at $1000 \mathrm{~mL} / \mathrm{h}$, and the total substitution fluid flow rate was $3000 \mathrm{~mL} / \mathrm{h}$. The substitution method consisted of both predilution and postdilution, with a median predilution substitution fluid flow rate of $2000 \mathrm{~mL} / \mathrm{h}$ and a median postdilution substitution fluid flow rate of $1000 \mathrm{~mL} / \mathrm{h}$. SMT mainly included rehydration, alkalinization of the urine with sodium bicarbonate, and appropriate diuresis. 
Table 1

Procedural characteristics of CVVHDF

\section{Category Product information}

\section{Device}

Equipment

Blood filter and extracorporeal circulation circuit

\section{Vascular access}

Type

Catheter

\section{Anticoagulants}

Drug

First dose (IU)

Additional dose (IU/h)

Liquid piercing

Blood flow rate $(\mathrm{mL} / \mathrm{min})$

Dialysate or substitution fluid

Dialysate flow rate $(\mathrm{mL} / \mathrm{h})$

Substitution method

Predilution fluid flow rate $(\mathrm{ml} / \mathrm{h})$

Postdilution fluid flow rate $(\mathrm{ml} / \mathrm{h})$

Duration of single treatment (h)

Number of CVVHDF sessions

1

2

3

Total number of treatments

Values are presented as median (25-75\% interquartile range) or number (percentage).

CVVHDF, continuous venovenous hemodiafiltration.

$3(33 \%)$

$2(23 \%)$

16
Baxter Prismaflex system (Meyzieu Cedex, France)

Baxter Prismaflex M150 set (Meyzieu Cedex, France)

Temporary catheter in right internal jugular vein or right femoral vein

Able $12 \mathrm{Fr}-16 / 20$-cm double-lumen central venous catheter (Foshan, Guangdong)

Low-molecular-weight heparin

$3000(2500-4750)$

$158(100-200)$

$0.9 \%$ sodium chloride injection $2000 \mathrm{~mL}$

$160(150-200)$

Qingshan Likang 4000 mL/bag (Chengdu, China)

1000

Combined predilution and postdilution

$2000(1000-2250)$

1000 (750-1500)

$16(10-22)$ 
Average number of treatments per

1.77 patient

Values are presented as median (25-75\% interquartile range) or number (percentage).

CVVHDF, continuous venovenous hemodiafiltration.

\subsection{Data collection}

The demographic, clinical, and laboratory data of the patients were collected at admission and during the treatment process, and included the following parameters: serum myoglobin (Mb), serum creatine kinase (CK), serum creatinine $(\mathrm{Cr})$, blood urea nitrogen $(\mathrm{BUN})$, serum potassium, and serum calcium. Renal survival data were collected for survival analysis; the terminal event for renal survival was an increase in the serum $\mathrm{Cr}$ level over the upper limit of the normal range. Additionally, data on hospitalization expenses and length of stay were collected for the health economic analysis. Data collection was mainly accomplished using the Yidu cloud intelligent data system (Yidu Cloud Corporation, Beijing, China), and part of the data was obtained from the original medical records.

\subsection{Selection of the control group}

We selected patients who were diagnosed with RM without AKI and treated using SMT only at the Inner Mongolia Medical University during the same period as the treatment-group patients. These patients were then matched to the treatment-group patients in a 1:1 ratio according to age, gender, etiology, complications, and laboratory test indexes on admission. The matched patients served as the control group for this study.

\subsection{Statistical analysis}

We used IBM SPSS Statistics 20.0 and GraphPad Prism v8.0 for data analysis and graph generation. Categorical variables were expressed as numbers and percentages, and continuous variables were expressed as medians and interquartile ranges (25-75\% quartiles), unless indicated otherwise. Variables were checked for normal distribution using the D'Agostino-Pearson omnibus normality test and the Shapiro-Wilk normality test. For comparisons, the chi-squared test, Mann-Whitney Utest, Wilcoxon matched-pairs signed rank test, and two-sided paired $t$ test were used, as appropriate. Renal survival in the two cohorts was analyzed using the Kaplan-Meier method and the log-rank test. All reported $P$ values are two-sided unless indicated otherwise, and $P<0.05$ was considered statistically significant.

\section{Results}

\subsection{Cohort characterization}

Between April 2017 and December 2020, 34 patients were diagnosed with RM and treated with CVVHDF and SMT in the Affiliated Hospital of Inner Mongolia Medical University. After the exclusion of patients 
with newly diagnosed AKI $(n=12)$, newly diagnosed coronary heart disease $(n=2)$, and a history of CKD $(n=11)$, a total of 9 patients with RM and without AKI who had been treated with CVVHDF combined with SMT were included in the treatment group. We then selected 9 control subjects who were matched in a 1:1 ratio to the treatment-group patients in terms of age, gender, cause of RM, leg and/or back pain, dark urine, history of hypertension, history of diabetes, history of heart disease, and biochemical index levels at admission. All control patients had been diagnosed with RM without AKI and received SMT only at the Inner Mongolia Medical University during the same period as the treatment-group patients. Figure 1 shows the flow chart for the selection of the patients enrolled in the two cohorts. The demographic and clinical details of the subjects are summarized in Table 2. 
Table 2

Demographic and clinical characteristics at admission

\begin{tabular}{|llll|}
\hline Variable & Treatment group $(\mathbf{n}=9)$ & Control group $(\mathbf{n}=9)$ & Pvalue \\
\hline Sex & & & 0.730 \\
\hline Male & $8(88.89 \%)$ & $2(22.22 \%)$ & 0.546 \\
\hline Age (years) & $1(11.11 \%)$ & & \\
\hline $10-19$ & & $2(22.22 \%)$ & \\
\hline $20-29$ & $2(22.22 \%)$ & $1(11.11 \%)$ & \\
\hline $30-39$ & $2(22.22 \%)$ & $2(22.22 \%)$ & \\
\hline $40-49$ & $0(0.00 \%)$ & $1(11.11 \%)$ & \\
\hline $50-59$ & $1(11.11 \%)$ & $1(11.11 \%)$ & \\
\hline $60-69$ & $0(0.00 \%)$ & $0(0.00 \%)$ & \\
\hline$\geq 70$ & $1(11.11 \%)$ & $2(22.22 \%)$ & \\
\hline Clinical symptoms & $3(33.33 \%)$ & & \\
\hline Myohemoglobinuria & & $2(22.22 \%)$ & \\
\hline Yes & $3(33.33 \%)$ & $3(33.33 \%)$ & \\
\hline No & $6(66.67 \%)$ & $6(66.67 \%)$ & \\
\hline Muscular soreness & & & \\
\hline Yes & & & \\
\hline No & $5(44.44 \%)$ & & \\
\hline Medical history & $5(55.56 \%)$ & & \\
\hline Hypertension history & & & \\
\hline Yes & & & \\
\hline No & & & \\
\hline
\end{tabular}

Treatment group, received continuous venovenous hemodiafiltration (CVVHDF) combined with standard medical therapy (SMT); control group, received SMT only. Values are presented as mean \pm standard deviation, median (25-75\% interquartile range), or number (percentage).

$\mathrm{Mb}$, myoglobin; $\mathrm{CK}$, creatine kinase; $\mathrm{CK}-\mathrm{MB}$, creatine kinase-MB; $\mathrm{Cr}$, creatinine; $\mathrm{BUN}$, blood urea nitrogen; $\mathrm{LDH}$, lactate dehydrogenase; $\mathrm{ALT}$, alanine aminotransferase; $\mathrm{AST}$, aspartate aminotransferase; WBC, white blood cell; HGB, hemoglobin; HCT, hematocrit; CO2-cp, carbon dioxide combining power; CTnT, cardiac troponin T; and AG, anion gap. 


\begin{tabular}{|c|c|c|c|}
\hline Variable & Treatment group $(n=9)$ & Control group $(n=9)$ & $P$ value \\
\hline Diabetes history & & & 0.730 \\
\hline Yes & $1(11.11 \%)$ & $0(0.00 \%)$ & \\
\hline No & $8(88.89 \%)$ & $9(100 \%)$ & \\
\hline Heart disease history & & & 0.730 \\
\hline Yes & $0(0.00 \%)$ & $0(0.00 \%)$ & \\
\hline No & $9(100 \%)$ & $9(100 \%)$ & \\
\hline \multicolumn{4}{|l|}{ Laboratory indicators } \\
\hline $\mathrm{Mb}(\mathrm{ng} / \mathrm{mL})$ & 3000 (2778.5-3000.0) & $2921(805.7-3000.0)$ & 0.796 \\
\hline CK (U/L) & $15,133(8881-123,845.5)$ & $17,155(8854.5-105,738.5)$ & 0.931 \\
\hline CK-MB (U/L) & 639.8 (149.4-1837.1) & $601.3(174.9-1859.3)$ & 0.796 \\
\hline $\mathrm{Cr}(\mu \mathrm{mol} / \mathrm{L})$ & $96.889 \pm 31.442$ & $67.556 \pm 9.002$ & 0.139 \\
\hline BUN (mmol/L) & $9.344 \pm 5.110$ & $5.967 \pm 2.588$ & 0.063 \\
\hline LDH (U/L) & $875(596.5-3773.5)$ & $616(550-2664.5)$ & 0.222 \\
\hline $\operatorname{ALT}(\mathrm{U} / \mathrm{L})$ & $85(55-356.3)$ & $109.5(82-268.2)$ & 0.931 \\
\hline AST (U/L) & $243(169-1222.0)$ & 300 (199-916.0) & 0.931 \\
\hline WBC $\left(10^{9} / L\right)$ & $15.898 \pm 7.391$ & $12.567 \pm 5.227$ & 0.147 \\
\hline $\mathrm{HGB}(\mathrm{g} / \mathrm{L})$ & $155.22 \pm 16.581$ & $151.22 \pm 7.563$ & 0.690 \\
\hline HCT (\%) & $45.6(41.5-52.8)$ & $43.5(41.6-46.4)$ & 0.796 \\
\hline $\mathrm{CO}_{2}-\mathrm{cp}(\mathrm{mmol} / \mathrm{L})$ & $20.433 \pm 6.464$ & $25.90 \pm 4.348$ & 0.153 \\
\hline Urine $(\mathrm{pH})$ & $5.5(5.3-5.8)$ & $6.5(5.5-7.3)$ & 0.063 \\
\hline $\mathrm{d}$-Dimer $(\mu \mathrm{g} / \mathrm{mL})$ & $1.77(0.5-4.2)$ & $0.6(0.2-0.8)$ & 0.136 \\
\hline CTnT (ng/mL) & $0.09(0.01-0.3)$ & $0.003(0.003-0.05)$ & 0.063 \\
\hline $\mathrm{Ca}^{2+}(\mathrm{mmol} / \mathrm{L})$ & $2.108 \pm 0.167$ & $2.103 \pm 0.169$ & 0.345 \\
\hline
\end{tabular}

Treatment group, received continuous venovenous hemodiafiltration (CVVHDF) combined with standard medical therapy (SMT); control group, received SMT only. Values are presented as mean \pm standard deviation, median (25-75\% interquartile range), or number (percentage).

$\mathrm{Mb}$, myoglobin; CK, creatine kinase; CK-MB, creatine kinase-MB; Cr, creatinine; $\mathrm{BUN}$, blood urea nitrogen; $\mathrm{LDH}$, lactate dehydrogenase; $\mathrm{ALT}$, alanine aminotransferase; $\mathrm{AST}$, aspartate aminotransferase; WBC, white blood cell; HGB, hemoglobin; HCT, hematocrit; CO2-cp, carbon dioxide combining power; CTnT, cardiac troponin T; and AG, anion gap. 


\begin{tabular}{|lccc|}
\hline Variable & Treatment group $(\mathbf{n}=9)$ & Control group $(\mathbf{n}=\mathbf{9})$ & Pvalue \\
\hline $\mathrm{K}^{+}(\mathrm{mmol} / \mathrm{L})$ & $4.4(4.2-4.5)$ & $4.3(2.8-4.5)$ & 0.796 \\
\hline $\mathrm{Na}^{+}(\mathrm{mmol} / \mathrm{L})$ & $138.400 \pm 4.410$ & $138.330 \pm 4.500$ & 0.919 \\
\hline $\mathrm{AG}(\mathrm{mmol} / \mathrm{L})$ & $16.967 \pm 11.507$ & $11.100 \pm 2.197$ & 0.177 \\
\hline $\begin{array}{l}\text { Treatment group, received continuous venovenous hemodiafiltration (CVVHDF) combined with } \\
\text { standard medical therapy (SMT); control group, received SMT only. Values are presented as mean } \pm \\
\text { standard deviation, median (25-75\% interquartile range), or number (percentage). }\end{array}$ \\
\hline $\begin{array}{l}\text { Mb, myoglobin; CK, creatine kinase; CK-MB, creatine kinase-MB; } \text { Cr, creatinine; BUN, blood urea } \\
\text { nitrogen; LDH, lactate dehydrogenase; ALT, alanine aminotransferase; AST, aspartate } \\
\text { aminotransferase; WBC, white blood cell; HGB, hemoglobin; HCT, hematocrit; CO2-cp, carbon dioxide } \\
\text { combining power; CTnT, cardiac troponin T; and AG, anion gap. }\end{array}$ \\
\hline
\end{tabular}

Of the 9 patients in the treatment group, 8 (88.89\%) patients were male. The age distribution of the patients in the treatment group was as follows: 10-19 years, $2(22.22 \%)$ patients; $20-29$ years, 2 (22.22\%) patients; $40-49$ years, 1 (11.11\%) patient; 60-69 years, 1 (11.11\%) patient; and $\geq 70$ years, 3 (33.33\%) patients. Muscular soreness was present in 4 (44.44\%) patients, and myohemoglobinuria was detected in $3(33.33 \%)$ patients. The laboratory test results in the treatment group were as follows: median serum Mb, $3000 \mathrm{ng} / \mathrm{mL}$ (2778.5-3000.0 ng/mL); median serum CK, 15,133 U/L (8881-123,845.5 $\mathrm{U} / \mathrm{L})$; median serum CK-MB, $639.8 \mathrm{U} / \mathrm{L}(149.4-1837.1 \mathrm{U} / \mathrm{L})$; serum $\mathrm{Cr}, 96.889 \pm 31.442 \mu \mathrm{mol} / \mathrm{L}$; and BUN, $9.344 \pm 5.110 \mathrm{mmol} / \mathrm{L}$.

Of the 9 patients in the control group, $7(77.78 \%)$ patients were male. Their age distribution was as follows: $10-19$ years, 2 (22.22\%) patients; $20-29$ years, 1 (11.11\%) patient; $30-39$ years, $2(22.22 \%)$ patients; $40-49$ years, $1(11.11 \%)$ patient; $50-59$ years, 1 (11.11\%) patient; and $\geq 70$ years, $2(22.22 \%)$ patients. Muscular soreness was present in $7(77.78 \%)$ patients, and myohemoglobinuria was detected in 3 (33.33\%) patients. In the control group, laboratory tests returned the following results: median serum Mb, 2921 ng/mL (805.7-3000.0 ng/mL); median serum CK, $17155 \mathrm{U} / \mathrm{L}$ (8854.5-105,738.5 U/L); median serum CK-MB, 601.3U/L (174.9-1859.3 U/L); mean serum Cr, $67.556 \pm 9.002 \mu \mathrm{mol} / \mathrm{L}$; and mean BUN, $5.967 \pm 2.588 \mathrm{mmol} / \mathrm{L}$. None of the above variables significantly differed between the two matched study groups.

\subsection{Comparisons between the treatment and control groups 3.2.1 Biochemical parameters}

Biochemical indicators were compared between the treatment and control groups before, and $24 \mathrm{~h}$ and 7 days after the first CVVHDF treatment (Figure 2). Some patients in the treatment group underwent 1 or 2 more CVVHDF treatment sessions after the first session. We found no significant between-group differences in any biochemical indicator at any time point, including serum $\mathrm{Mb}$, serum $\mathrm{CK}$, serum $\mathrm{Cr}$, BUN, 
serum lactate dehydrogenase (LDH), serum aspartate aminotransferase (AST), serum carbon dioxide combining power $\left(\mathrm{CO}_{2}-\mathrm{cp}\right)$, serum calcium, serum potassium, serum sodium, and serum anion gap (AG).

\subsubsection{Renal survival}

All patients were followed up from hospital admission to January, 2021. The follow-up duration ranged from 53 to 4 months. One patient in the treatment group died of acute myocardial infarction 12 months after hospital discharge. No other patient developed increased serum $\mathrm{Cr}$ (i.e., the terminal event) during follow-up. Renal survival analysis with the log-rank test showed no significant difference between the treatment and control groups $(P=1.000$; Figure 3$)$.

\subsubsection{Health economics}

The indicators of health economics were compared between the treatment and control groups (Figure 4). The total cost ( $6446.70 \pm 3184.45$ vs. $2482.60 \pm 2354.42$ US dollars, $P=0.008)$ and average daily cost (744.87 $\pm 335.68 v s .232 .45 \pm 228.37$ US dollars, $P=0.002$ ) during hospitalization were significantly higher in the treatment group than in the control group. Moreover, the duration of hospitalization did not significantly differ between the treatment and control groups $(8.78 \pm 1.99 \mathrm{vs} .12 .22 \pm 4.76$ days, $P=$ 0.063).

\subsection{Comparisons within the treatment group}

\subsubsection{Biochemical indicators before and after the first CVVHDF treatment}

The comparison of biochemical indicators before and after the first CVVHDF treatment in the treatment group is shown in Figure 5. After the first CVVHDF treatment, the levels of serum Mb, serum Cr, BUN, serum sodium, and white blood cells (WBCs) significantly decreased, while the serum $\mathrm{CO}_{2}-\mathrm{cp}$ significantly increased compared to the pretreatment levels. Other indicators did not significantly change after the first CVVHDF session.

\subsubsection{Biochemical indicators at different time points}

The comparison of the biochemical indicators on admission and at 1, 2, and 7 days after admission in the treatment group is shown in Figure 6. We found significant downward trends in the levels of serum $\mathrm{Mb}$, serum $\mathrm{CK}$, serum $\mathrm{Cr}, \mathrm{BUN}$, and WBCs; no trend was found in the other indicators.

\section{Discussion}

To our knowledge, the present retrospective cohort study is the first to compare the effectiveness of CVVHDF combined with SMT vs. SMT alone in preventing the occurrence of AKI in patients with RM and 
to analyze the related health costs. Our findings demonstrate that although a single session of CVVHDF could facilitate myoglobin elimination, its addition to SMT did not significantly improve the serum Mb level or the levels of other biochemical indices or the long-term renal prognosis. However, the addition of CVVHDF to SMT did greatly increase the hospitalization costs.

While the therapeutic effects of RRT on RM-induced AKI have been extensively studied, its usefulness as a preventive measure is yet to be determined. Currently available blood-purification techniques, such as high-flux hemodialysis, hemofiltration, hemodiafiltration, and plasmapheresis, can effectively eliminate myoglobin, which is a small protein molecule with a molecular weight of $17 \mathrm{kDa}$. Some studies have investigated the capacity of these blood-purification techniques to eliminate myoglobin. Sorrentino et al. measured the myoglobin clearance in 6 patients with RM-induced AKI, and found that high-flux hemodialysis effectively eliminated myoglobin, with a median myoglobin clearance of $90.5 \mathrm{~mL} / \mathrm{min}$ (range, $52.4-126.3 \mathrm{~mL} / \mathrm{min}$ ) and a median myoglobin removal per treatment hour of $0.54 \mathrm{~g}$ (range, $0.15-$ $2.21 \mathrm{~g}$ )[11]. Naka et al. reported the case of a 53-year-old woman with RM and AKI for whom hemofiltration resulted in a myoglobin clearance of $30.5-39.2 \mathrm{~mL} / \mathrm{min}$ and removed $0.55-0.64 \mathrm{~g}$ myoglobin/treatment hour for 8 hours of treatment[12]. In a case series of 6 patients with RM-induced AKI, the mean myoglobin clearance was $81 \mathrm{~mL} / \mathrm{min}$ (range, 42-131 $\mathrm{mL} / \mathrm{min}$ ) after hemodiafiltration with a postdilutional fluid substitution rate of $2-3 \mathrm{~L} / \mathrm{h}[13]$. A control study with a two-stage crossover design also verified that hemodiafiltration could effectively clear myoglobin[14]. Although we did not find any studies that confirmed that plasmapheresis eliminates myoglobin, we speculate that this is definitely the case because during plasmapheresis, plasma is non-selectively discarded and replaced with exogenous fresh plasma.

However, none of the above studies indicate whether these therapeutic measures can prevent the occurrence of RM-induced AKI because effective myoglobin clearance does not directly indicate a good prognosis[15]. Hence, cohort studies or randomized controlled trials with good control groups and renal survival data are required to directly investigate the ability of blood-purification techniques to prevent RMinduced AKI. We were unable to find any such studies via a literature search. We did find a systematic review that investigated the therapeutic effects of continuous renal replacement therapy (CRRT) for RM and RM-induced AKI[16]. This review included 4 studies from China, one of which contained patients without AKI and the other 3 studies contained patients with AKI. The review concluded that although CRRT may provide some benefits for RM patients, the poor methodological quality of the included studies and the lack of data on clinically important outcomes meant that there was insufficient evidence to discern any likely benefits of CRRT over conventional therapy for the prevention of RM-induced AKI[16]. A case report found that plasmapheresis did not prevent renal failure in a patient with RM-induced AKI[17]. We found no other study that explored the protective effects of plasmapheresis against RM-induced AKI. However, several studies have explored the therapeutic effects of plasmapheresis on RM-induced AKI and found that this treatment was effective[18, 19]. Nevertheless, as these studies were case reports or case analyses without a control group, Szpirt considered that the use of plasmapheresis was not justified for the treatment of RM and AKI[20]. 
CVVHDF is the most common CRRT technique, and combines hemofiltration and hemodialysis, so it could effectively clear both middle-molecular-weight urotoxins and micromolecular urotoxins, including myoglobin. Therefore, we conducted this retrospective cohort study to determine whether CVVHDF combined with the SMT vs. SMT only could prevent RM-induced AKI. Our study showed that although a single CVVHDF treatment facilitated myoglobin elimination, compared with SMT only, CVVHDF combined with SMT did not significantly improve the serum Mb levels or other biochemical indices or the long-term renal prognosis of patients with RM. Thus, we consider that the available clinical evidence does not show any benefit of hemodiafiltration to prevent RM-induced AKI.

Interestingly, an animal experiment was performed to explore the direct renal protective effect of continuous venovenous hemofiltration (CVVH) in the early stage of RM[21]. In this study, the 2 hind legs of mongrel dogs were intramuscularly injected with $50 \%$ hypertonic glycerol to establish RM, and $2 \mathrm{~h}$ after the injection, $\mathrm{CVVH}$ was performed for $8 \mathrm{~h}$. The study confirmed that at the cellular and molecular levels, $\mathrm{CVVH}$ treatment mitigated myoglobin-induced mitochondrial damage by inhibiting the mitochondrial apoptotic pathway and cell apoptosis; the treatment also delayed the occurrence of oliguria and protected the renal function during the early stage of RM development[21]. However, we consider that this single animal experiment cannot accurately represent the actual clinical condition due to the following reasons: First, in clinical practice, patients generally receive blood-purification treatments 1-2 days or even longer after the occurrence of RM, rather than being treated $2 \mathrm{~h}$ after the pathogenic onset like in the animal experiment; by the time blood-purification treatment is initiated, myoglobin has already caused some renal damage at the molecular level. Second, the CRRT regimen in most dialysis centers is 6-12 $\mathrm{h}$ daily or every other day for several days, so the observation period should be longer; however, in the animal experiment, observations were performed before and after a single treatment. Third, even with extracorporeal circulation therapy, the kidneys are still the main organs responsible for removing myoglobin.

In our study, we also conducted a health economic analysis, and found that although the total duration of hospitalization did not differ between the treatment and control groups, both the total and daily hospitalization costs were significantly higher in the former than in the latter. Although few studies conduct health economic analyses, the cost of healthcare is a problem that cannot be ignored. CRRT, especially, is an expensive treatment requiring more medical insurance funds and patients' financial resources, especially in less-developed areas.

The present study has certain limitations. First, this was a retrospective study with a small sample size. More large-scale prospective studies are required to explore this issue, including cohort studies and randomized controlled trials. Second, the ability of high-flux hemodialysis and plasmapheresis to prevent RM-induced $\mathrm{AKI}$ is also worth exploring.

\section{Conclusion}


In summary, although a single CVVHDF treatment could facilitate myoglobin elimination, CVVHDF combined with SMT as compared to SMT only did not significantly improve the serum Mb levels or other biochemical indices or the long-term renal prognosis. Although the total hospitalization duration did not differ between the two groups, CVVHDF combined with SMT obviously increased the total and daily hospitalization costs.

\section{Abbreviations}

$A G$

anion gap

AKI

acute kidney injury

AST

aspartate aminotransferase

BUN

blood urea nitrogen

CK

creatine kinase

CKD

chronic kidney disease

$\mathrm{Cr}$

creatinine

CRRT

continuous renal replacement therapy

$\mathrm{CVVH}$

continuous venovenous hemofiltration

CVVHDF

continuous venovenous hemodiafiltration

$\mathrm{CO}_{2}$-cp

carbon dioxide combining power

ICU

intensive care unit

$\mathrm{LDH}$

lactate dehydrogenase

$\mathrm{Mb}$

myoglobin

RM

rhabdomyolysis

RRT

renal replacement therapy 
SMT

standard medical therapy

WBC

white blood cell

\section{Declarations}

\section{Acknowledgments}

The abstract of this article was presented by post in the Blood Purification Forum of the Chinese Society of Nephrology in 2021.

\section{Authors' Contributions}

YM, DS, and JZ were responsible for the study concept and design. YM, MZ, CW, and DW were responsible for the acquisition, analysis, and interpretation of the data. YM and JZ were responsible for drafting the manuscript. MZ was responsible for the statistical analysis. All authors had full access to all the data in the study and take responsibility for the integrity of the data and the accuracy of the data analysis. All authors interpreted the findings, contributed to writing the manuscript, and approved the final version for publication. All authors read and approved the final manuscript.

\section{Funding}

This work was supported by the National Natural Science Foundation of China (nos., 81960143, 81960130), the Program for Young Talents of Science and Technology in Universities of Inner Mongolia Autonomous Region (no., NJYT-19-B34), the "Going far" Talent Program of Inner Mongolia Medical University (no., ZY0130015), the Trinity College Students Innovation and Entrepreneurship Cultivation Project of Inner Mongolia Medical University (no., SWYT2020008), the Head Start Program of the Affiliated Hospital of Inner Mongolia Medical University (no., FYQMJH2020026), and the Project of Hebei Provincial Health and Family Planning Commission (no., 20181078).

\section{Availability of data and materials}

Data are available on request.

\section{Ethics approval and consent to participate}

The study was approved by the ethics committee of the Affiliated Hospital of Inner Mongolia Medical University (no., WZ2021029) and performed in accordance with the ethical standards laid down in the 1964 Declaration of Helsinki and its later amendments. The investigators verbally informed the patients or their legal representatives about the study, and obtained written informed consent before the participants' entry into the study. 
Not applicable.

\section{Competing interests}

The authors declare that they have no competing interests.

\section{References}

1. Chavez LO, Leon M, Einav S, et al. Beyond muscle destruction: a systematic review of rhabdomyolysis for clinical practice. Critical Care. 2016; 20(1):1-11.

2. Cabral BMI, Edding SN, Portocarrero JP, et al. Rhabdomyolysis. Disease-a-Month. 2020; 66(8):1-18.

3. Huerta-Alardín A, Varon J, Marik PE. Bench-to-bedside review: Rhabdomyolysis - an overview for clinicians. Critical Care. 2005; 9(2):158-169.

4. Delaney KA, Givens ML, Vohra RB. Use of RIFLE criteria to predict the severity and prognosis of acute kidney injury in emergency department patients with rhabdomyolysis. Journal of Emergency Medicine. 2012; 42(5):521-528.

5. Chatzizisis YS, Misirli G, Hatzitolios Al, et al. The syndrome of rhabdomyolysis: Complications and treatment. European Journal of Internal Medicine. 2008; 19(8):568-574.

6. Bosch X, Poch E, Grau JM. Rhabdomyolysis and acute kidney injury. New England Journal of Medicine. 2009; 361(14):62-72.

7. Petejova N, Martinek A. Acute kidney injury due to rhabdomyolysis and renal replacement therapy: a critical review. Crit Care. 2014; 18(3):224-231.

8. Naka T, Jones D, Baldwin I, et al. Myoglobin clearance by super high-flux hemofiltration in a case of severe rhabdomyolysis: a case report. Crit Care. 2005; 9(2):R90-95.

9. Premru V, Kovač J, Buturović-Ponikvar J, et al. High cut-off membrane hemodiafiltration in myoglobinuric acute renal failure: a case series. Ther Apher Dial. 2011;15(3):287-291.

10. Kidney Disease Improving Global Outcomes (KDIGO). Acute Kidney Injury Work Group: KDIGO clinical practice guideline for acute kidney injury. Kidney international Supplement. 2012; 2(1):1-138.

11. Sorrentino SA, Kielstein JT, Lukasz A, et al. High permeability dialysis membrane allows effective removal of myoglobin in acute kidney injury resulting from rhabdomyolysis. Critical Care Medicine. 2011; 39(1):184-186.

12. Naka T, Jones D, Baldwin I, et al. Myoglobin clearance by super high-flux hemofiltration in a case of severe rhabdomyolysis: a case report. Critical Care. 2005; 9(2):1-6.

13. Premru V, Kova J, Buturovi-Ponikvar J, et al. High Cut-off Membrane Hemodiafiltration in Myoglobinuric Acute Renal Failure: A Case Series. Therapeutic Apheresis and Dialysis. 2011; 15(3):287-291.

14. Peltonen $S$, Åhlström A, Kylävainio V, et al. The effect of combining intermittent hemodiafiltration with forced alkaline diuresis on plasma myoglobin in rhabdomyolysis. Acta Anaesthesiologica Scandinavica. 2010; 51(5):553-558. 
15. Cruz DN, Bagshaw SM. Does continuous renal replacement therapy have a role in the treatment of rhabdomyolysis complicated by acute kidney injury? Semin Dial. 2011; 24(4):417-420.

16. Zeng X, Zhang L, Wu T, et al. Continuous renal replacement therapy (CRRT) for rhabdomyolysis. Cochrane Database Syst Rev. 2014; 15(6):1-40.

17. Cornelissen JJ, Haanstra W, Haarman HJ, et al. Plasma exchange in rhabdomyolysis. Intensive Care Med. 1989; 15(8):528-529.

18. Swaroop R, Zabaneh R, Parimoo N. Plasmapheresis in a patient with rhabdomyolysis: a case report. Cases J. 2009; 2:8138.

19. Maccario M, Fumagalli C, Dottori V, et al. The association between rhabdomyolysis and acute renal failure in patients undergoing cardiopulmonary bypass. J Cardiovasc Surg (Torino). 1996; 37(2):153-159.

20. Szpirt WM. Plasmapheresis is not justified in treatment of rhabdomyolysis and acute renal failure. J Cardiovasc Surg (Torino). 1997; 38(5):557.

21. Tang W, Chen Z, Wu W, et al. Renal protective effects of early continuous venovenous hemofiltration in rhabdomyolysis: improved renal mitochondrial dysfunction and inhibited apoptosis. Artif Organs. 2013; 37(4):390-400.

\section{Figures}




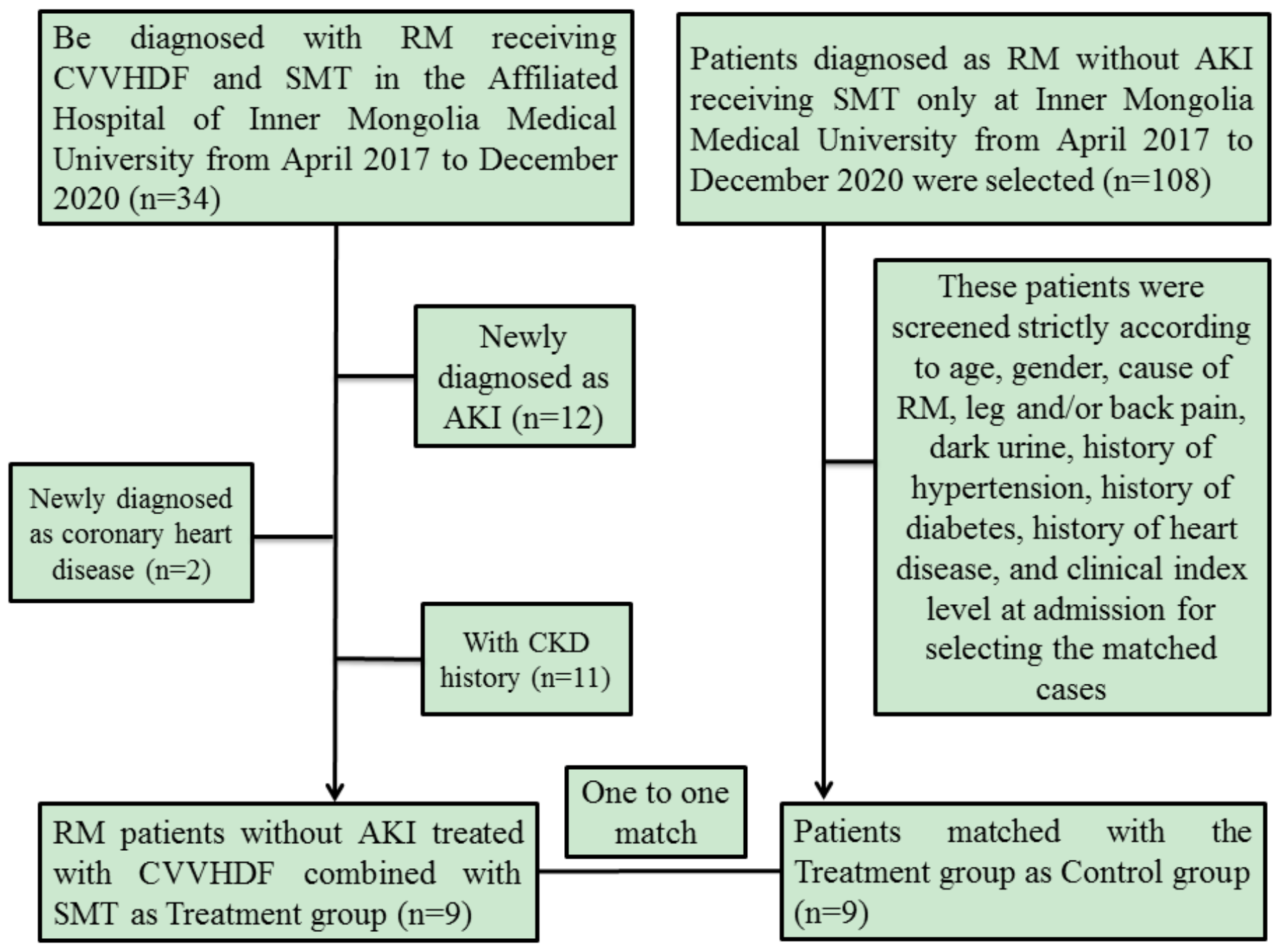

Figure 1

Flow chart of study participants. RM, rhabdomyolysis; CVVHDF, continuous venovenous hemodiafiltration; SMT, standard medical therapy; AKI, acute kidney injury; CKD, chronic kidney disease. 
A

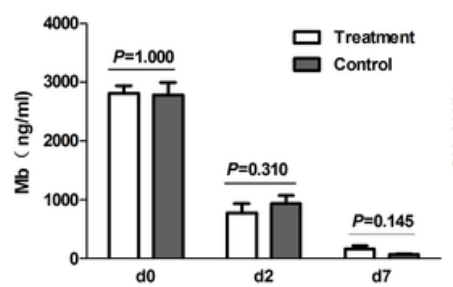

$\mathbf{E}$

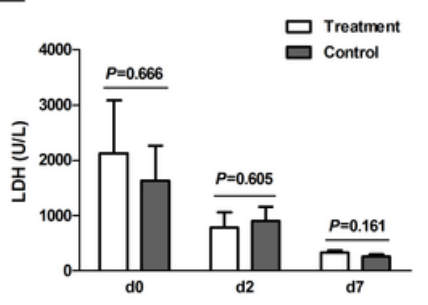

I

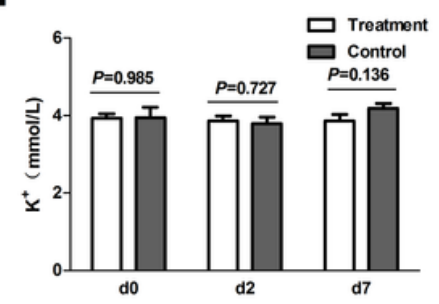

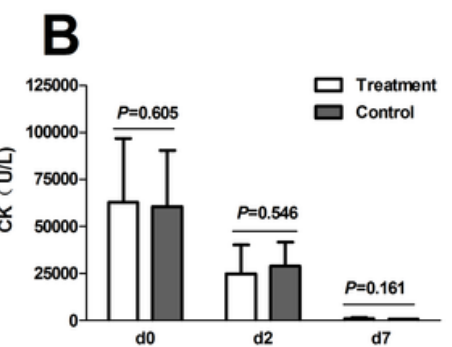
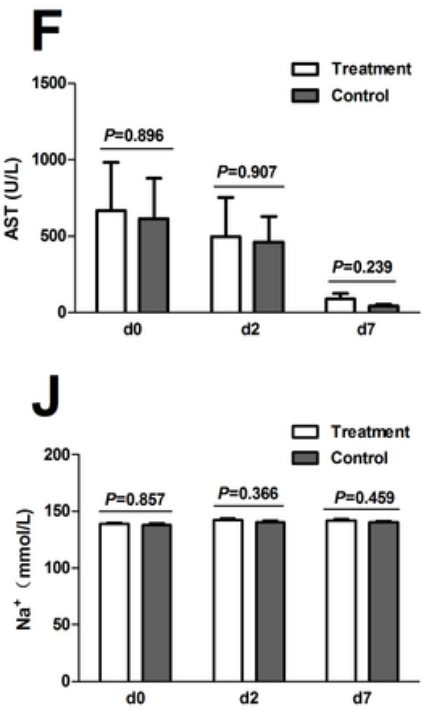

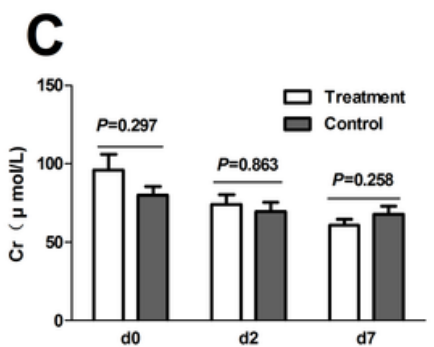

\section{G}
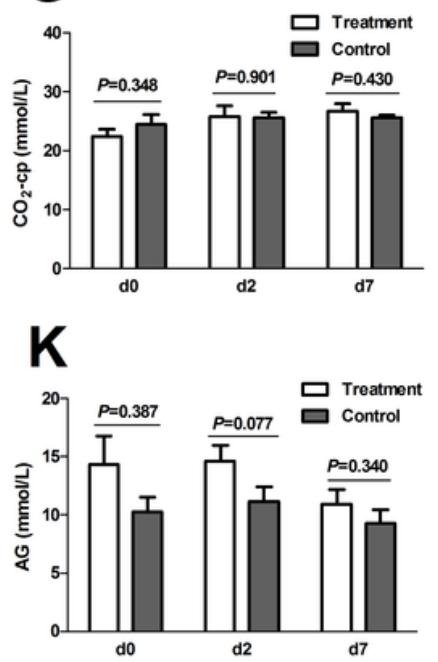
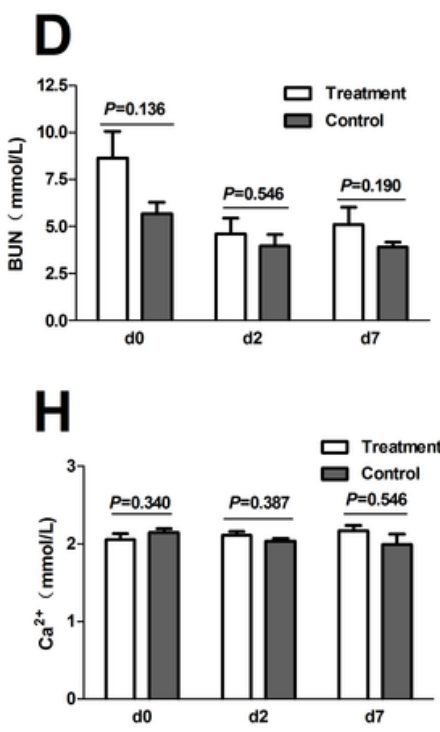

Figure 2

Comparison of biochemical indicators between the treatment group and control group at different time points. Treatment group, received continuous venovenous hemodiafiltration (CVVHDF) combined with standard medical therapy (SMT); control group, received SMT only. D0, before the first CVVHDF treatment; $\mathrm{d} 2$ and d7, $24 \mathrm{~h}$ and 7 days after the first CVVHDF treatment. Some patients received 1 or 2 more treatment(s) after the first treatment. Mb, myoglobin; $\mathrm{CK}$, creatine kinase; $\mathrm{Cr}$, creatinine; BUN, blood urea nitrogen; LDH, lactate dehydrogenase; AST, aspartate aminotransferase; CO2-cp, carbon dioxide combining power; $A G$, anion gap. 


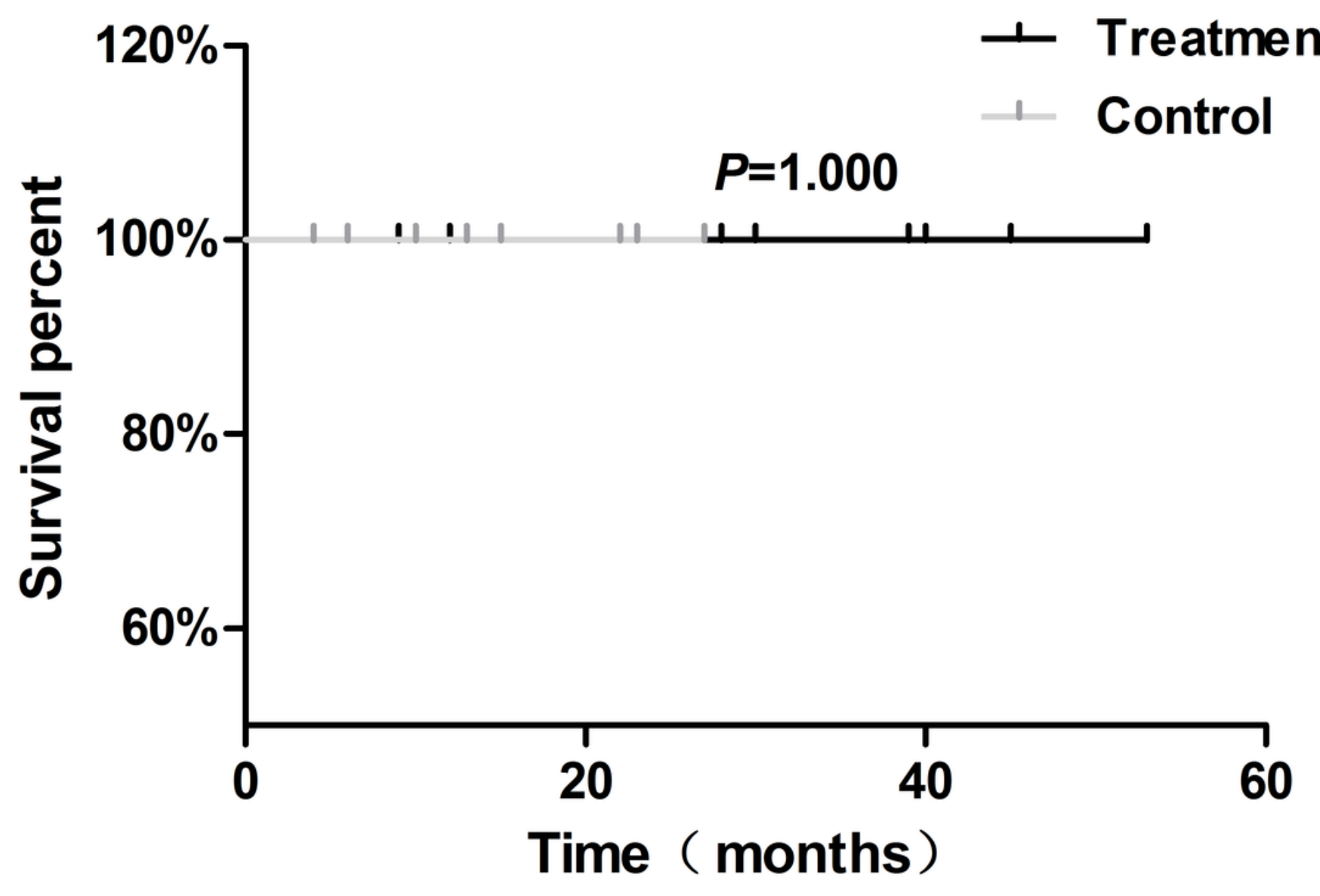

Figure 3

Comparison of renal survival rate after 53 months between the treatment group and control group. Treatment group, received continuous venovenous hemodiafiltration combined with standard medical therapy (SMT); control group, received SMT only.
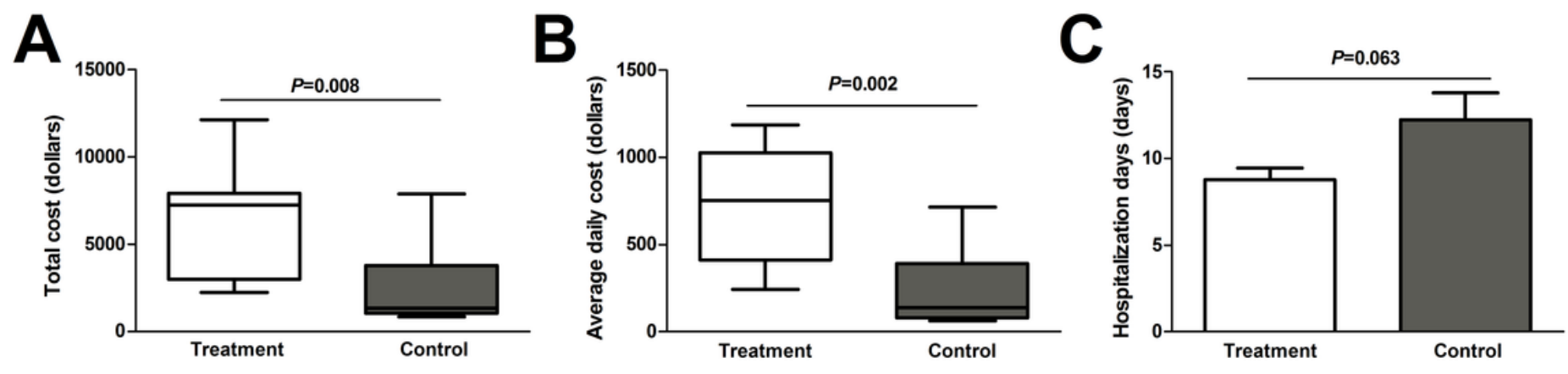

Figure 4

Comparison of health economic indexes between the treatment group and control group. Treatment group, received continuous venovenous hemodiafiltration combined with standard medical therapy 
(SMT); control group, received SMT only.

A
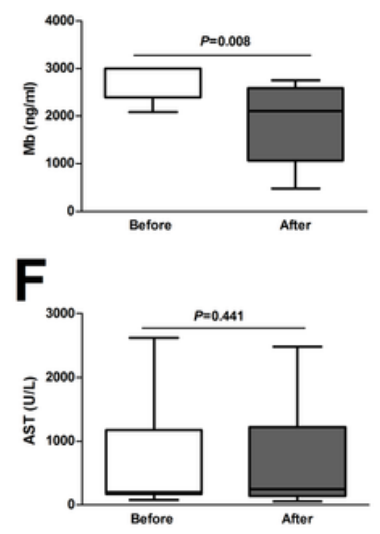

K

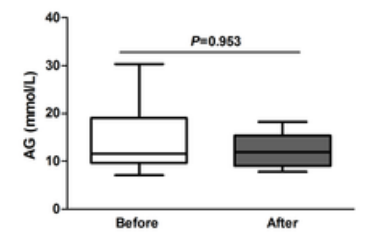

B
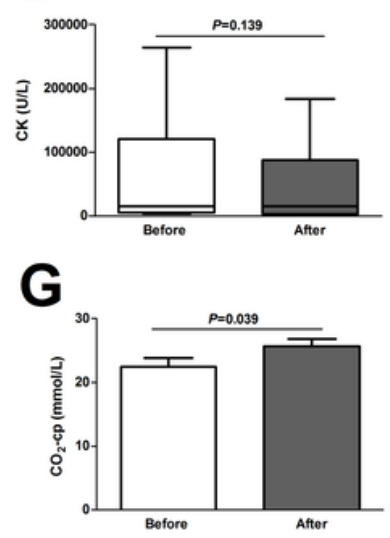

$\mathbf{L}$

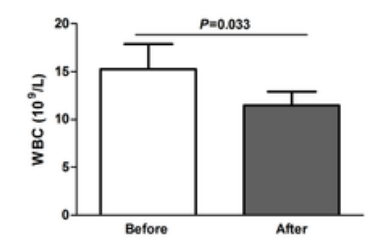

C

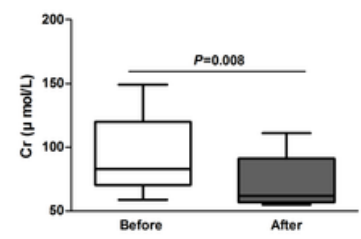

H

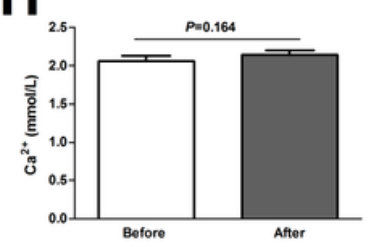

M

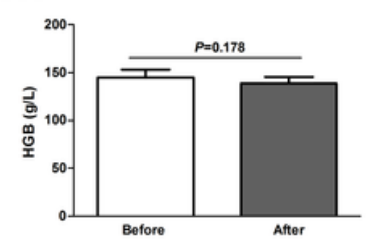

D $\quad E$
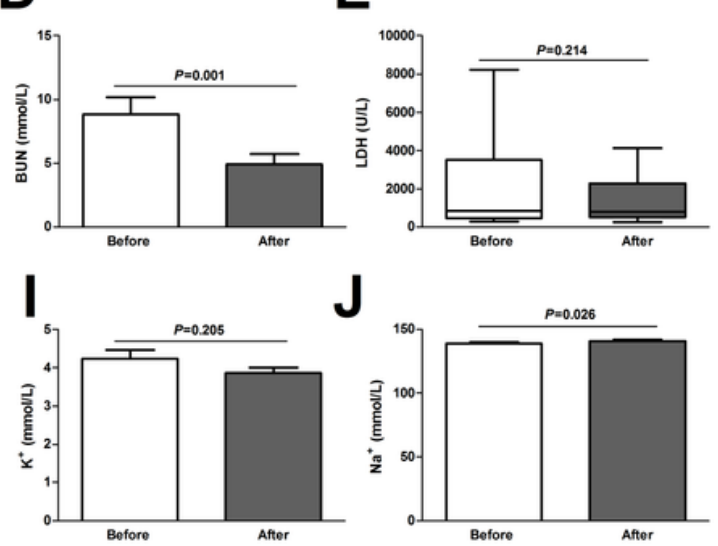

\section{Figure 5}

Comparison of biochemical indicators before and after the first continuous venovenous hemodiafiltration (CVVHDF) treatment in the treatment group. Treatment group, received CVVHDF combined with standard medical therapy. $\mathrm{Mb}$, myoglobin; $\mathrm{CK}$, creatine kinase; $\mathrm{Cr}$, creatinine; $\mathrm{BUN}$, blood urea nitrogen; $\mathrm{LDH}$, lactate dehydrogenase; AST, aspartate aminotransferase; $\mathrm{CO}$-cp, carbon dioxide combining power; $\mathrm{AG}$, anion gap; WBC, white blood cell; HGB, hemoglobin. 

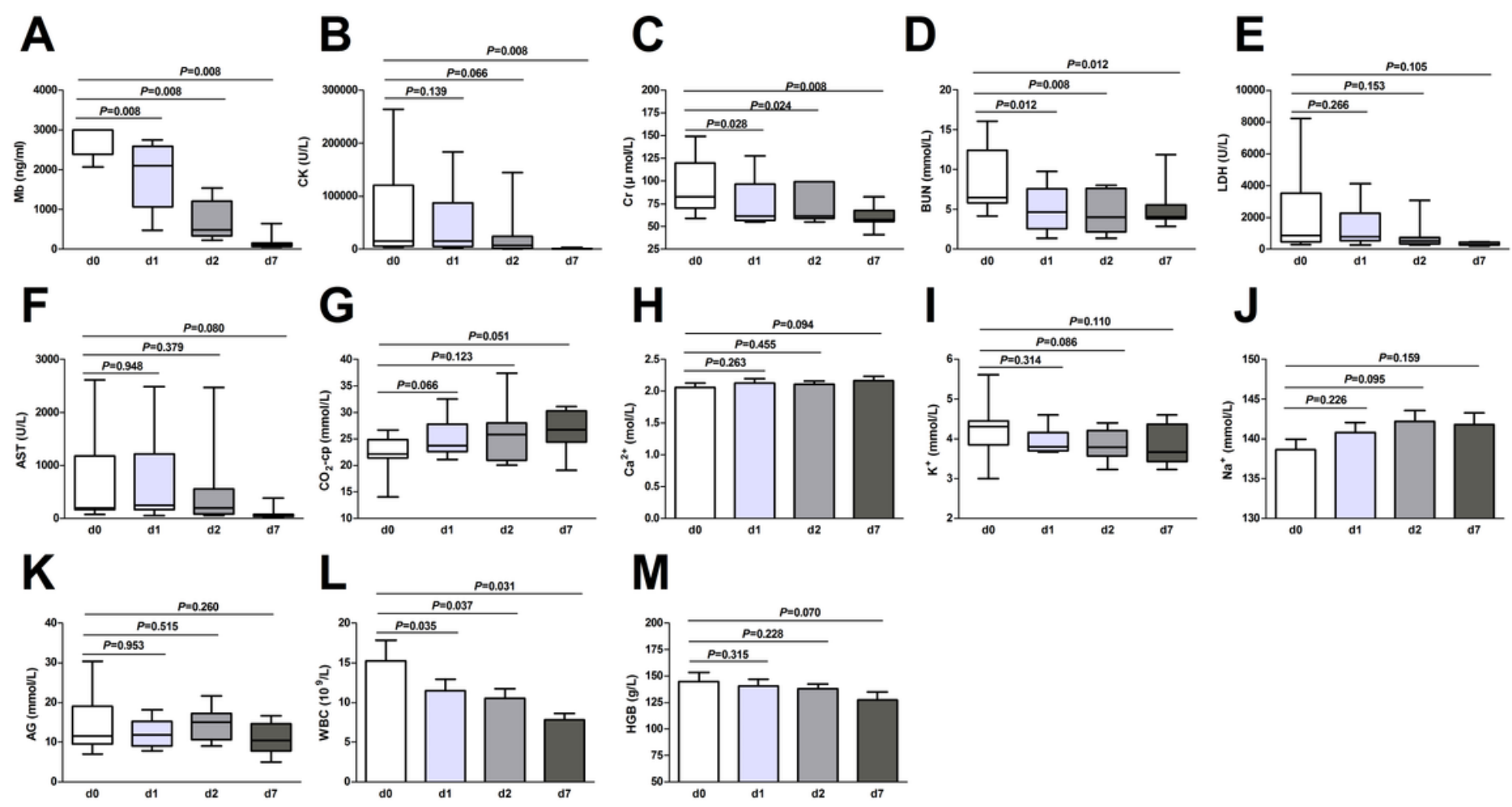

Figure 6

Comparison of biochemical indicators at different time points in the treatment group. Treatment group, received continuous venovenous hemodiafiltration combined with standard medical therapy. D0, on admission; d1, d2 and d7, 1 day, 2 days, and 7 days after admission. Mb, myoglobin; CK, creatine kinase; $\mathrm{Cr}$, creatinine; BUN, blood urea nitrogen; $\mathrm{LDH}$, lactate dehydrogenase; AST, aspartate aminotransferase; CO2-cp, carbon dioxide combining power; AG, anion gap; WBC, white blood cell; HGB, hemoglobin. 